\title{
Low Coronal Signatures of a Coronal Mass Ejection
}

\author{
K. P. Qiu, Y. Dai, and Y. H. Tang \\ Department of Astronomy, Nanjing University, Nanjing, 210093, P. R. China, \\ email: kpqiu@nju.edu.cn
}

\begin{abstract}
Using the observations of the EUV Imaging Telescope(EIT) and the Large Angle Spectrometric Coronagraph(LASCO) on the Solar and Heliospheric observatory $(S O H O)$ and solar soft X-ray flux and radio bursts data, we study the low coronal signatures of a solar limb coronal mass ejection(CME) on November 4, 2003. The two prominent dimmings in EIT difference images were closely related to two large loops in this event. The onset time and height of the CME and the lower limit of the masses loss from dimming regions are estimated.
\end{abstract}

Keywords. Sun: corona, Sun: coronal mass ejections, Sun: flares, Sun: UV radiation

\section{Introduction}

Solar coronal dimmings observed in soft X-ray (See for example Sterling \& Hundson 1997) and EUV (see for example Zarro et al. 1999; Delannée, Delaboudinière \& Lamy 2000) are expected to be the very first signature of CMEs. Sometimes large coronal loops disappearing are associated to CMEs (see for example Delannée \& Aulanier 1999). Here we study the EUV dimmings and loop structures of the 4 November 2003 event.

\section{Observations}

The great X28/3B flare occurred at 19:29 UT on 2003 November 4 in NOAA active region 10486 was associated to a fast halo CME. Observations of the EUV Imaging Telescope(EIT) and the Large Angle Spectrometric Coronagraph(LASCO) on the Solar and Heliospheric observatory $(S O H O)$ and GOES soft X-ray flux and radio bursts data are used.

Figure 1 shows the large loops (denoted as 'L1' and 'L2') with one ends anchored in the flaring region (denoted as ' $F$ ') in EIT $195 \AA$ and $284 \AA$, at 19:35 UT and 19:05 UT respectively. L1's height is about $0.35 R_{\odot}$, or $2.5 \times 10^{5} \mathrm{~km}$, from the solar surface. The loops disappeared in the next EIT $195 \AA$ image at 19:47 UT and no longer appeared. In Figure 2 the EIT difference images by subtracting the preflare image at 19:25 UT show two dimmings (denoted as ' $\mathrm{d} 1$ ' and 'd2') at 19:47 UT, 19:59 UT and 20:11 UT. It is clear that $\mathrm{d} 1$ and $\mathrm{d} 2$ correspond to the footpoints of L1 and L2. A lower limit of the preflare masses from dimming regions is estimated to be about $6.6 \times 10^{15} \mathrm{~g}$ and this is consistent with typical CME masses. The first CME observation of LASCO/C2 at 19:54 UT co-aligned with the EIT observation( the middle image in Figure 2) is shown in Figure 3. It can be seen that the double EUV dimmings approximately correspond to the CME footpoints. Using the CME velocity of $\sim 2300 \mathrm{kms}^{-1}$ inferred from the linear fit of LASCO observations we extrapolate that the CME time at the height of L1 was 19:44 UT. This is well in the loops disappearing time window of 19:35 UT - 19:47 UT.

From above observations we suggest that the CME initiated as the large loops expansion or opening, apparently disappearing, at about 19:44 UT. Figure 4 shows that the inferred CME onset time (denoted as the vertical line) is in the rising phase of the soft 

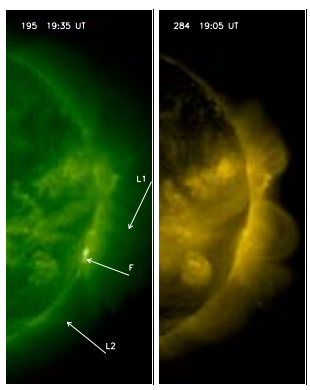

Figure 1. Large loops in EIT $195 \AA$ and $284 \AA$ channels

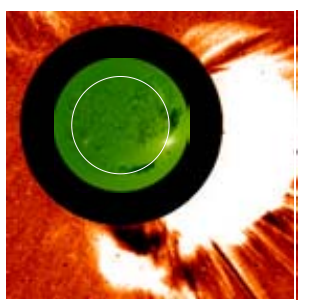

Figure 3. Co-alignment of $\mathrm{LASCO} / \mathrm{C} 2$ and EIT $195 \AA$ observations

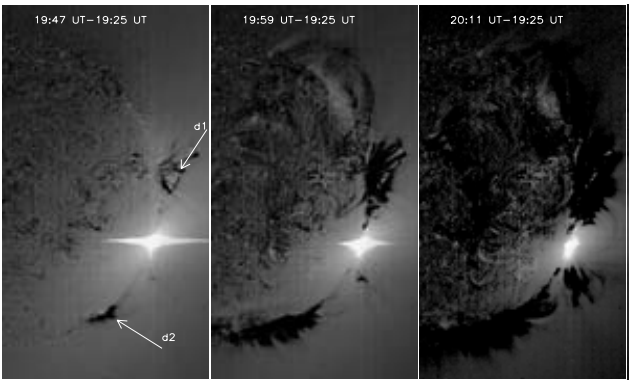

Figure 2. Double dimmings evolution in EIT $195 \AA$ Aifference images

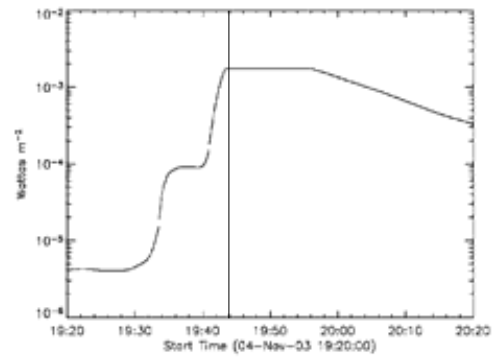

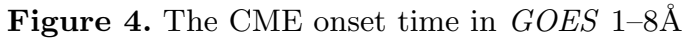
soft X-ray flux

X-ray flare. The moving type IV radio burst (from Solar Geophysical Data) started at 19:47 UT and it is just after the CME onset time of 19:44 UT. According to plasma emission mechanism, the upper frequency of the burst $180 \mathrm{MHz}$ gives a height of $0.38 R_{\odot}$ under the corona density model of Sittler \& Guhathakurta (1999) and this is consistent with the loops height or the CME onset height of $0.35 R_{\odot}$.

\section{Conclusions}

According to the observations and some estimations, we suggest that the CME initiated as the large loops expansion or opening, leaving behind two prominent dimmings, in the rising phase of the soft X-ray flare.

\section{Acknowledgements}

SOHO is a project of international cooperation between ESA and NASA. This work is Supported by NKBRSF (G2000078404) and NSFC (No.10073005).

\section{References}

Delannée, C. \& Aulanier, G. 1999 Sol. Phys. 190, 107

Delannée, C., Delaboudinière, J.-P. \& Lamy, P. 2000 A\&A 355, 725

Sittler, E.C. \& Guhathakurta, M. 1999 ApJ 523, 812

Sterling, A.C. \& Hudson, H.S. 1997 ApJ (letters) 491, L55

Zarro, D.M., Sterling, A.C., Thompson, B.J., Hudson, H.S., \& Nitta, N. 1999 ApJ (letters) 520, L139 